\title{
RAIRO
}

ANALYSE NUMÉRIQUE

\section{Philippe CORTEY-DumONT}

\section{Approximation numérique d'une inéquation quasi variationnelle liée à des problèmes de gestion de stock}

RAIRO - Analyse numérique, tome 14, no 4 (1980), p. 335-346.

<http://www.numdam.org/item?id=M2AN_1980_14_4_335_0>

(C) AFCET, 1980, tous droits réservés.

L'accès aux archives de la revue «RAIRO - Analyse numérique » implique l'accord avec les conditions générales d'utilisation (http://www.numdam.org/ legal.php). Toute utilisation commerciale ou impression systématique est constitutive d'une infraction pénale. Toute copie ou impression de ce fichier doit contenir la présente mention de copyright.

\section{Numdam}




\title{
APPROXIMATION NUMÉRIQUE D'UNE INÉQUATION QUASI VARIATIONNELLE \\ LIĖE A DES PROBLĖMES \\ DE GESTION DE STOCK $\left({ }^{*}\right)$
}

\author{
par Philippe Cortey-Dumont $\left({ }^{1}\right)$ \\ Communiqué par P -A RAVIART
}

Résumé - On étudie l'approximation par éléments fins d'inéquations quası vartatıonnelles apparaissant en contrôle impulsionnel On démontre que l'ordre de convergence est en $h|\log h|$ sans restrictions sur la dimension du domaine

Abstract - We study the approximation by a finite element method of quast-variational inequalities related to impulse control We show that the order of convergence is in $h|\log h|$ without conditions for the dimension of the domain

On s'intéresse à l'approximation par une méthode d'élément fini conforme de l'inéquation quasi variationnelle suivante (en abrégé IQV) issue de problème de contrôle impulsionnel [5].

Trouver $u \in H^{1}(\Omega)$ solution de :

$$
a(u, v-u) \geqq(f, v-u), \quad v \leqq M u, \quad v \in H^{1}(\Omega),
$$

où

$$
M u(x)=1+\inf u(x+\xi), \quad \xi \geqq 0, \quad x+\xi \in \Omega .
$$

Si on appelle $u_{h}$ la solution de l'IQV discrète, on montre sans condition de dimension :

1) que sous des hypothèses de régularıté $W^{2, p}$ :

$$
\begin{gathered}
\left\|u-u_{h}\right\|_{\infty} \leqq C h|\log h|, \\
\left\|u-u_{h}\right\|_{1} \leqq C(h|\log h|)^{1 / 2} ;
\end{gathered}
$$

$\left(^{*}\right)$ Reçu jullet 1979 , révisé avril 1980

(1) L A N I, Faculté des Sciences, 25030 Besançon Cedex

R A I R O Analyse numérıque/Numerical Analysıs, 0399-0516/1980/335/\$ 500

(C) Bordas-Dunod 
2) que sous des hypothèses de régularité $W^{1, \infty}$ :

$$
\begin{aligned}
& \lim _{h \rightarrow 0}\left\|u-u_{h}\right\|_{\infty}=0, \\
& \lim _{h \rightarrow 0}\left\|u-u_{h}\right\|_{1}=0 .
\end{aligned}
$$

On améliore ainsi les résultats de [10] (lim $\left\|u-u_{h}\right\|_{\infty}=0$ en dimension 2); de [15] ( $\left.\underline{\lim } u_{h}=u\right)$; de [29] $\left(\left\|u-u_{h}\right\|_{\infty} \leqq C h|\log h|\right.$ en dimension 1).

\section{MÉTHODE}

Le peu de propriété de continuité de l'opérateur qui défınit les contraintes ne permet pas d'exploiter l'estimation a priori classique $\left\|u_{h}\right\|_{H^{1}(\Omega)}<C$ et d'utiliser au niveau de l'IQV la notion de convexe « corlsistant-convergeant » (cf. [14]) ou convergence au sens de Mosco (cf. [31]).

Il a donc semblé préférable d'introduire l'algorithme constructif qui a permis à Bensoussan-Lıom el le n-lème ltéré de sa version discrète en fonctıon de l'erreur continu. L'étape principale de l'étude est alors l'obtention d'une estimation de l'écart, en norme uniforme, entre le $n$-ième itéré de l'algorithme classique de Bensoussan-Lions et le $n$-ième itéré de sa version discrète, en fonction de l'erreur commise, én norme uniforme, lors de l'approximation par élément fini des $n$ premières inéquations intervenant dans la version continue de l'algorithme évoqué ci-dessus $(c f .[11])$.

L'article se décompose en deux parties : au paragraphe I on introduit les notations et on fait quelques rappels; le paragraphe II est consacré aux résultats et à leurs démonstrations.

\section{POSITION DU PROBLĖME}

Soit $\Omega$ un polygone de $\mathbb{B}^{N}$ sur lequel nous ferons ultérieurement quelques hypothèses de régularité $[c f .(6),(7),(8),(9)]$.

On munit $H^{1}(\Omega)$ de sa structure d'ordre usuelle et on introduit la forme bilinéaire $a(u, v)$ suivante :

$$
a(u, v)=\int_{\Omega}\left(\sum_{i j=1}^{N} a_{i j} \frac{\partial u}{\partial x_{i}} \frac{\partial v}{\partial x_{j}}+\sum_{i=1}^{N} b_{i} \frac{\partial u}{\partial x_{i}} v+a_{0} u v\right) d x
$$

On suppose :

(1) $a(u, v)$ continue, fortement coercive $\left(\exists \alpha>0\right.$ tel que $\left.\alpha\|v\|^{2} \leqq a(v, v)\right)$. De plus, les coefficients $a_{i j}, b_{i}, a_{0}$ sont réguliers et $a_{0} \geqq \mu>0$. 
(2) Soit $f \in L^{\infty}(\Omega) ; f \geqq 0 ;\|f\|_{\infty}>\mu$.

On considère alors un opérateur $M$ de $L^{\infty}(\Omega)$ dans lui-même défini comme suit :

$$
M u(x)=1+\inf u(x+\xi), \quad \xi \geqq 0, \quad x+\xi \in \Omega .
$$

Ceci nous permet alors de définir l'IQV suivante :

Trouver $u \in H^{1}(\Omega)$ solution de :

$$
\left\{\begin{array}{c}
a(u, v-u) \geqq(f, v-u), \quad v \leqq M u, \quad v \in H^{1}(\Omega), \\
u \leqq M u .
\end{array}\right.
$$

Remarque : On a supposé $\mu<\|f\|_{\infty}$ pour éviter que l'IQV ne se réduise à l'équation $a\left(\hat{u}_{0}, v\right)=(f, v), v \in H^{1}(\Omega)$.

Notation et définition : On note $\sigma(\psi)$ la solution de l'inéquation variationnelle suivante :

$$
\left\{\begin{array}{c}
a(\sigma(\psi), v-\sigma(\psi)) \geqq(f, v-\sigma(\psi)), \quad v \leqq \psi, \quad v \in H^{1}(\Omega) \\
\sigma(\psi) \leqq \psi .
\end{array}\right.
$$

DériNition: On introduit l'algorithme suivant : partant de :

$$
u_{0} \in \mathbb{C}(\bar{\Omega}) \cap H^{1}(\Omega) .0 \leqq u_{0} \leqq \hat{u}_{0} .
$$

(4) On pose $u_{n}=\sigma\left(M u_{n-1}\right), n=1,2, \ldots$

(5) On note $\hat{u}_{n}$ et $\breve{u}_{n}$ les itérés obtenus par cet algorithme à partir de $\hat{u}_{0}$ et de $\check{u}_{0}=0$.

RÉGULARITÉ SUR L'OUVERT : On suppose :

(6) si $\psi \in \mathbb{C}(\bar{\Omega})$ alors $\sigma(\psi) \in \mathbb{C}(\bar{\Omega})$;

(7) si $\psi \in W^{2, p}(\Omega), \quad \partial \psi / \partial v=0$ et $f \in L^{p}(\Omega)$ alors $\sigma(\psi) \in W^{2, p}(\Omega)$ et $\partial \sigma(\psi) / \partial v=0$;

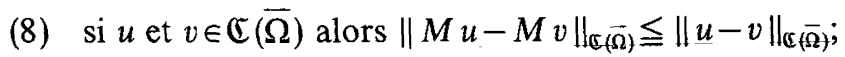

(9) si $u \in W^{1, \infty}(\Omega)$ alors $\|M u\|_{W^{1, \infty}(\Omega)} \leqq C\|u\|_{W^{1, \infty}(\Omega)}$.

QUelQues RAPPELS : Sous ces conditions, on sait :

(10) qu'il existe une solution au problème (3) ( $c f .[4,5,24])$;

(11) qu'elle est unique ( $c f .[18]$ );

(12) qu'elle est continue et que $\left\|u-u_{n}\right\|_{\mathbb{C}(\bar{\Omega})} \leqq \lambda^{n}\left\|\hat{u}_{0}\right\|_{\mathbb{E}(\bar{\Omega})}$ avec $\lambda=1-\mu /\|f\|_{\infty}$ (cf. [17], on peut également se reporter à [19] pour une autre estimation du comportement de l'algorithme). 
LA DISCRÉTISATION : On se propose de discrétiser $H^{1}(\Omega)$ en utilisant une méthode d'éléments finis conformes. Le peu de régularité de la solution de l'IQV $u$ et de l'obstacle $M u$ semblent déconseiller l'utilisation de polynômes de degré supérieur à 1 .

On établit sur $\bar{\Omega}$ une triangulation $\mathfrak{I}_{h}$ composée d'un nombre fini de $N$-simplexes non dégénérés telle que :

$-\bar{\Omega}=U T_{h}$;

- $\stackrel{\circ}{T} \cap \stackrel{\circ}{T^{\prime}}=\emptyset$;

- pour chaque simplexe, toute face est la face d'un autre simplexe ou un morceau de la frontière $\Gamma$.

On note $h(T)$ le diamètre de $T$ et $\rho(T)$ le sup des diamètres des sphères inscrites. On suppose que pour tout $T \in \mathfrak{T}_{h}$ :

$$
0<\hat{v} \leqq \frac{h(T)}{\rho(T)} \leqq v .
$$

On appelle :

$$
V_{h}=\left\{v_{h} \in \mathbb{C}(\bar{\Omega}) \cap H^{1}(\Omega) \text { tel que } v_{h} \text { soit linéaire sur tout simplexe } T\right\} .
$$

Soit $M_{i} i \in I=\{1, \ldots, m(h)\}$ un sommet de la triangulation, on note par $\varphi_{i}$ la fonction de $V_{h}$ qui vérifie $\varphi_{i}\left(M_{j}\right)=\delta_{i j}$. On sait alors que les fonctions $\varphi_{i}$ avec $i \in I$ forment une base de l'espace $V_{h}$.

On définit l'opérateur de restriction comme suit :

$$
\text { pour } \quad v \in \mathbb{C}(\bar{\Omega}) \cap H^{1}(\Omega), \quad r_{h} v(x)=\sum_{i \in I} v\left(M_{i}\right) \varphi_{i}(x) .
$$

Rappelons maintenant quelques propriétés élémentaires de ce type d'approximation :

$$
\varphi_{i}(x) \geqq 0, \quad \forall i \in I \quad \text { et } \quad \sum_{i \in I} \varphi_{i}(x)=1 .
$$

Soit $v_{h}(x)=\sum_{i \in I} v_{i} \varphi_{i}(x)$ et $u_{h}(x)=\sum_{i \in I} u_{i} \varphi_{i}(x)$ :

$$
\begin{gathered}
\text { si } \quad v_{h} \geqq 0 \quad \text { alors } \forall i \in I, \quad v_{i} \geqq 0, \\
\left\|u_{h}-v_{h}\right\|_{\mathbb{C}(\bar{\Omega})}=\underset{i \in I}{\operatorname{Max}\left|u_{i}-v_{i}\right|,} \\
\left\|r_{h} u-r_{h} v\right\|_{\mathbb{C}(\bar{\Omega})} \leqq\|u-v\|_{\mathbb{C}(\bar{\Omega})} .
\end{gathered}
$$


On définit alors l'IQV discrète associée au problème (3).

On cherche donc $u_{h} \in V_{h}$ solution de :

$$
\left\{\begin{array}{c}
a\left(u_{h}, v_{h}-u_{h}\right) \geqq\left(f, v_{h}-u_{h}\right), \quad v_{h} \leqq r_{h} M u_{h}, \\
u_{h} \leqq r_{h} M u_{h} .
\end{array}\right.
$$

LA MATRICE DE DISCRÉTISATION : On introduit de manière naturelle la matrice de discrétisation $A$ de coefficient générique $a_{i j}=a\left(\varphi_{i}, \varphi_{j}\right)$. On identifiera à $\eta$ le vecteur constant de $\mathbb{R}^{m(h)}$ de composante égale à $\eta$. Enfin si $v=\left(v_{1}, \ldots, v_{m(h)}\right)$ est un vecteur de $\mathbb{R}^{m(h)}$ on posera :

$$
[A v]_{i}=\sum_{j \in I} a_{i j} v_{j}
$$

On suppose :

$$
a_{i j}=a\left(\varphi_{i}, \varphi_{j}\right) \leqq 0 \quad \text { pour } \quad i \neq j .
$$

On trouvera dans [9] des conditions géométriques simples pour lesquelles (19) se trouve vérifié.

LEMME : Sous les hypothèses (2)-(19), pour tout vecteur $\eta>0$, on $a: A \eta>0$. En particulier $A$ est une $M$-matrice.

En effet, d'après (14) $[A \eta]_{i}=\eta \int_{\Omega} a_{0}(x) d x>0$.

Ce lemme nous permet d'affirmer l'existence et l'unicité d'une solution au problème $(18)$.

NotATION ET DÉfINITION : Soit $\psi \in \mathfrak{C}(\bar{\Omega})$, on note $\sigma_{h}(\psi)$ la solution de l'inéquation variationnelle discrète suivante :

$$
\left\{\begin{array}{c}
a\left(\sigma_{h}(\psi), v_{h}-\sigma_{h}(\psi)\right) \geqq\left(f, v_{h}-\sigma_{h}(\psi)\right), \\
\sigma_{h}(\psi) \leqq r_{h} \psi
\end{array}\right.
$$

DÉfInItion : On définit l'algorithme discret suivant : partant de $u_{0 h} \in V_{h}$ : (20) $\left(\hat{u}_{0 h}\right.$ est la solution de $\left.a\left(\hat{u}_{0 h}, v_{h}\right)=\left(f, v_{h}\right)\right)$, on pose $u_{n h}=\sigma_{h}\left(M u_{n-1 h}\right)$; (21) on note $\hat{u}_{n h}$ et $\check{u}_{n h}$ les itérés obtenus par cet algorithme à partir de $\hat{u}_{0 h}$ et de $\check{u}_{0 h}=0$;

(22) enfin, on posera : $w_{n h}=\sigma_{h}\left(M u_{n-1}\right)$ et $w_{0 h}=u_{0 h}, \hat{w}_{0 h}=\hat{u}_{0 h}, \check{w}_{0 h}=0$.

Nous sommes maintenant en mesure d'étudier le comportement de la suite $\left\{u_{h}\right\}$ quand $h$ tend vers zéro (dans tout ce qui suit, $C$ désignera une constante non déterminée indépendante de $h$ ). 


\section{RESULTATS}

Énonçons le résultat suivant (on renvoit à [12] pour des démonstrations dans le cas discret et à [30] pour une utilisation dans le cas continu).

(23) Soit $\psi(h), \tilde{\psi}(h) \in \mathbb{C}(\bar{\Omega})$.

Proposition 1 : Sous les hypothèses (1), (2), (19), (23), on a :

$$
\left\|\sigma_{h}(\psi(h))-\sigma_{h}(\widetilde{\psi}(h))\right\|_{\mathbb{G}(\bar{\Omega})} \leqq\|\psi(h)-\widetilde{\psi}(h)\|_{\mathbb{G}(\bar{\Omega})} .
$$

Abordons maintenant le lemme suivant qui s'avère essentiel pour l'obtention des résultats.

LEMME : Sous les hypothèses (1), (2), (6), (8), (19), pour tout $n$, on a :

(25) $\left\|u-u_{h}\right\|_{\mathbb{E}(\bar{\Omega})} \leqq \sum_{i=0}^{n}\left\|\check{u}_{i}-\check{w}_{i h}\right\|_{\mathbb{Q}(\bar{\Omega})}$

$$
\left\|u_{n}-u_{n h}\right\|_{\mathbb{E}(\bar{\Omega})} \leqq \sum_{i=0}^{n}\left\|u_{i}-w_{i h}\right\|_{\mathbb{C}(\bar{\Omega})}
$$

$$
+2 \sum_{i=0}^{n}\left\|\hat{u}_{i}-\hat{w}_{i h}\right\|_{\mathbb{G}(\bar{\Omega})}+\left\|u-\check{u}_{n}\right\|_{\mathbb{C}(\bar{\Omega})}+2\left\|u-\hat{u}_{n}\right\|_{\mathbb{G}(\bar{\Omega})},
$$

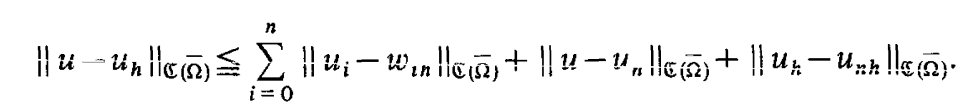

Démontrons par récurrence (24) :

- pas 1: $u_{1}=\sigma\left(M u_{0}\right), u_{1 h}=\sigma_{h}\left(M w_{0 h}\right), w_{1 h}=\sigma_{h}\left(M u_{0}\right)$. On applique la proposition 1 avec $\psi(h)=M u_{0}, \widetilde{\psi}(h)=M w_{0 h}$, ce qui est possible d'après (4) et (8). Donc, en utilisant de nouveau (8) :

$$
\left\|u_{1 h}-w_{1 h}\right\|_{\mathbb{E}(\bar{\Omega})} \leqq\left\|M w_{0 h}-M u_{0}\right\|_{\mathbb{S}(\bar{\Omega})} \leqq\left\|w_{0 h}-u_{0}\right\|_{\mathbb{G}(\bar{\Omega})} .
$$

Soit :

$$
\left\|u_{1}-u_{1 h}\right\|_{\mathbb{C}(\bar{\Omega})} \leqq\left\|u_{1}-w_{1 h}\right\|_{\mathbb{G}(\vec{\Omega})}+\left\|w_{1 h}-u_{1 h}\right\|_{\mathbb{G}(\bar{\Omega})} \leqq \sum_{i=0}^{1}\left\|u_{i}-w_{i h}\right\|_{\mathbb{E}(\vec{\Omega})}
$$

et $M u_{1} \in \mathbb{C}(\bar{\Omega})$ d'après $(8)$.

- pas $n$ : on sait donc par hypothèse que $M u_{n-1} \in \mathbb{C}(\bar{\Omega})$ et que :

$$
\left\|u_{n-1}-u_{n-1 h}\right\|_{\mathbb{C}(\bar{\Omega})} \leqq \sum_{i=0}^{n-1}\left\|u_{i}-w_{i h}\right\|_{\mathbb{E}(\bar{\Omega})} .
$$


On applique alors la proposition 1 avec $\psi(h)=M u_{n-1}$ et $\tilde{\psi}(h)=M u_{n-1 h}$. Donc, en utilisant (8) :

$$
\left\|u_{n h}-w_{n h}\right\|_{\mathbb{C}(\bar{\Omega})} \leqq\left\|M u_{n-1}-M u_{n-1 h}\right\|_{\mathbb{G}(\bar{\Omega})} \leqq\left\|u_{n-1}-u_{n-1 h}\right\|_{\mathbb{C}(\bar{\Omega})} .
$$

Ainsi :

$$
\left\|u_{n}-u_{n h}\right\|_{\mathbb{E}(\bar{\Omega})} \leqq \sum_{i=0}^{n}\left\|u_{i}-w_{i h}\right\|_{\mathbb{G}(\bar{\Omega})}
$$

et d'après (18) $M u_{n} \in \mathbb{C}(\bar{\Omega})$.

Démontrons maintenant (25) :

$$
\left\|u-u_{h}\right\|_{\mathbb{C}(\bar{\Omega})} \leqq\left\|u-\hat{u}_{n}\right\|_{\mathbb{C}(\bar{\Omega})}+\left\|\hat{u}_{n}-\hat{u}_{n h}\right\|_{\mathbb{E}(\bar{\Omega})}+\left\|\hat{u}_{n h}-u_{h}\right\|_{\mathbb{C}(\bar{\Omega})},
$$

mais $\check{u}_{n h} \leqq u_{h} \leqq \hat{u}_{n h}$. Donc en utilisant la normalité de $\mathfrak{C}(\bar{\Omega})$ :

$$
\left\|u-u_{h}\right\|_{\mathbb{C}(\bar{\Omega})} \leqq\left\|u-\hat{u}_{n}\right\|_{\mathbb{E}(\bar{\Omega})}+\left\|\hat{u}_{n}-\hat{u}_{n h}\right\|_{\mathbb{C}(\bar{\Omega})}+\left\|\hat{u}_{n h}-\check{u}_{n h}\right\|_{\mathbb{C} \bar{\Omega})} \text { d'où }(25) \text {. }
$$

Enfin (26) découle immédiatement de (24).

$$
\text { C.Q.F.D. }
$$

Il apparaît ainsi de manière "naturelle » que l'approximation d'une IQV elliptique stationnaire peut être ramenée par l'énoncé précédent à l'étude de la convergence uniforme de l'approximation d'une IV. Ce problème a été résolu dans le cas $H_{0}^{1}(\Omega)$ par Baiocchi [1] et Nitsche [22].

On considère :

$$
\begin{aligned}
\psi \in W^{1, \infty}(\Omega), & \|\psi\|_{W^{1, \infty}}<C, \\
\sigma(\psi) \in W^{2, p}(\Omega), & \|\sigma(\psi)\|_{W^{2, p}}<C .
\end{aligned}
$$

On note $\overline{\sigma_{h}(\psi)}$ la solution de :

$$
a\left(\overline{\sigma_{h}(\psi)}, v_{h}\right)=a\left(\sigma(\psi), v_{h}\right), \quad \forall v_{h} \in V_{h} .
$$

On suppose :

$$
\left\|\sigma(\psi)-\overline{\sigma_{h}(\psi)}\right\|_{\mathbb{E}(\bar{\Omega})} \leqq C h .
$$

On pourra se reporter à [23] pour vérifier (30), voir également [22].

Proposition 2 : Sous les hypothèses (1), (2), (19), (27), (28), (30) on a :

$$
\left\|\sigma(\psi)-\sigma_{h}(\psi)\right\|_{\mathbb{C}(\bar{\Omega})} \leqq C h .
$$

vol. $14, n^{\circ} 4,1980$ 
On a développé la démonstration de [22].

HyPothÈSE DE RÉGUlarité : On suppose :

$$
\left\{\begin{array}{cc} 
& \operatorname{Max}_{n}\left\{\left\|\hat{u}_{n}\right\|_{W^{2},},\left\|\check{u}_{n}\right\|_{W^{2} n},\|u\|_{W^{2, p}}\right\} \leqq C \\
\text { et } & \forall n \quad \frac{\partial \hat{u}_{n}}{\partial v}=\frac{\partial \check{u}_{n}}{\partial v}=\frac{\partial u}{\partial v}=0 .
\end{array}\right.
$$

Hannouzet-Joly [16] et Caffarelli-Friedman [7] ont donné des conditions pour que l'hypothèse (31) soit vérifiée ( $c f$. aussi U. Mosco [32]).

THÉORÈME 1 : Sous les hypothèses (1), (2), (6) à (9), (19), (30), (31), pour tout n, on $a$ :

$$
\begin{gathered}
\left\|u-u_{h}\right\|_{\mathfrak{C}(\bar{\Omega})} \leqq C h|\log h|, \\
\left\|u-u_{h}\right\|_{H^{1}(\Omega)} \leqq C(h|\log h|)^{1 / 2} .
\end{gathered}
$$

Démonstration : D'après (12) $\left\|u-\hat{u}_{n}\right\|_{\mathbb{C}(\overline{\Omega)}} \leqq C \lambda^{n}\left\|u-\check{u}_{n}\right\|_{\mathbb{C}(\bar{\Omega})} \leqq C \lambda^{n}$.

On applique la proposition 2 ce qui est possible d'après (9) et (31). Donc, $\forall n$ :

$$
\left\|\hat{u}_{n}-\hat{w}_{n h}\right\|_{\mathbb{E}(\bar{\Omega})} \leqq C h\left\|\check{u}_{n}-\check{w}_{n h}\right\|_{\mathbb{C}(\bar{\Omega})} \leqq C h .
$$

L'estimation (25) du lemme nous donne donc :

$$
\left\|u-u_{h}\right\|_{\mathbb{E}(\bar{\Omega})} \leqq C\left(\lambda^{n}+n h\right) .
$$

Suivant Loinger [29], on pose $h=\lambda^{n}$.

On reprend maintenant l'estimation de [13], [33] ce qui est possible d'après (31). Donc :

$$
\left\|u-u_{h}\right\|_{H^{\prime}(\Omega)}^{2} \leqq C\left(\left\|u-v_{h}\right\|_{H^{1}(\Omega)}^{2}+\left\|u-v_{h}\right\|_{L^{2}(\Omega)}+\left\|u_{h}-v\right\|_{L^{2}(\Omega)}\right),
$$

pour tout $v \leqq M u$ et tout $v_{h} \leqq r_{h} M u_{h}$.

On a d'après (9) :

$$
\left\|M u-M u_{h}\right\|_{\mathfrak{C}(\bar{\Omega})} \leqq\left\|u-u_{h}\right\|_{\mathbb{Q}(\bar{\Omega})} \leqq C h|\log h| .
$$

On prend alors $v_{h}=r_{h} u-C h|\log h| \leqq r_{h} M u_{h}$ donc :

$$
\begin{array}{r}
\left\|u-v_{h}\right\|_{H^{1}(\Omega)} \leqq C(h|\log h|+h), \\
\left\|u-v_{h}\right\|_{L^{2}(\Omega)} \leqq C\left(h|\log h|+h^{2}\right) .
\end{array}
$$


On pose ensuite $v=\inf \left(u_{h}, M u\right) \leqq M u$ et on obtient $\left|u_{h}-v\right| \leqq\left|r_{h} M u_{h}-M u\right|$ p.p. donc

$$
\left\|u_{h}-v\right\|_{L^{2}(\Omega)} \leqq C(h|\log h|+h) .
$$

On reporte (33) (34) (35) dans (32) pour obtenir le résultat.

Remarque : On peut retrouver dans le cas discret l'estimation (12) de Hannouzet-Joly [17] et montrer que pour

$$
0 \leqq u_{0 h} \leqq \hat{u}_{0 h}, \quad\left\|u_{n h}-u_{h}\right\|_{\mathbb{C}(\bar{\Omega})} \leqq \lambda^{n}\left\|\hat{u}_{0 h}\right\|_{\mathbb{E}(\bar{\Omega})} \leqq \lambda^{n}\|f\|_{\infty} .
$$

Ceci permet d'obtenir le théorème 1 à partir de (26).

La connaissance de la surface libre est très intéressante pour déterminer la stratégie à suivre. Déduisons du théorème 1 le corollaire suivant. On pose :

$$
\begin{gathered}
S=\{x \in \Omega \text { tel que } u(x)<M u(x)\}, \\
\tilde{S}_{h}=\left\{x \in \Omega \text { tel que } u_{h}(x)<M u_{h}(x)-h^{1 / 2}|\log h|\right\} .
\end{gathered}
$$

Corollaire: Sous les hupothèses (1), (2), (6) à (9), (19), (30), (31), on a :

$$
\lim _{h \rightarrow 0} \tilde{S}_{h}=S \quad \text { (au sens des ensembles) et } \tilde{S}_{h} \subset S \text { pour } h \text { petit. }
$$

Le résultat du théorème $1\left\|u-u_{h}\right\|_{\mathbb{C}(\bar{\Omega})} \leqq C h|\log h|$ permet d'appliquer une démonstration de Baiocchi-Pozzi [3].

$\mathrm{Vu}$ les difficultés rencontrées pour vérifier l'hypothèse (31), il peut être intéressant d'indiquer un résultat de convergence sous des hypothèses de régularité plus faibles.

On suppose :

(36) pour tout $\psi \in U^{1},(\Omega)$ et tout $\varepsilon>0$, il existe $\psi_{1} \in W^{2 \cdot}{ }^{\prime \prime}(\Omega)$ tel que $\partial \psi_{\varepsilon} / \partial v=0$ et $\left\|\psi-\psi_{\varepsilon}\right\|_{\mathbb{(}(\bar{\Omega})}<\varepsilon$. (on trouvera dans [10] des conditions suffisantes pour que (36) ait lieu):

(37) on suppose mantenant : $\sigma(\psi) \in W^{1,}(\Omega)$.

Proposition 3 : Sous les hypothèses (1), (2), (7), (9), (19), (27), (30), (36), (37), on $a$ :

$$
\lim _{h \rightarrow 0}\left\|\sigma(\psi)-\sigma_{h}(\psi)\right\|_{\mathbb{(}(\bar{\Omega})}=0
$$


D'après la version continue de la proposition 1 , on a :

$$
\left\|\sigma(\psi)-\sigma\left(\psi_{\varepsilon}+\varepsilon\right)\right\|_{(\bar{\Omega}) \leqq} \leqq 2 \varepsilon \quad \text { et } \quad\left\|\sigma(\psi)-\sigma\left(\psi_{\varepsilon}-\varepsilon\right)\right\|_{\mathbb{G} \bar{\Omega} !} \leqq 2 \varepsilon .
$$

De plus, $\forall h>0, \sigma_{h}\left(\psi_{\varepsilon}-\varepsilon\right) \leqq \sigma_{h}(\psi) \leqq \sigma_{h}\left(\psi_{\varepsilon}+\varepsilon\right)$.

Or, d'après la proposition 2 :

$$
\lim _{h \rightarrow 0}\left\|\sigma\left(\psi_{\varepsilon}+\varepsilon\right)-\sigma_{h}\left(\psi_{\varepsilon}+\varepsilon\right)\right\|_{\mathbb{C}(\bar{\Omega})}=\lim _{h \rightarrow 0}\left\|\sigma\left(\psi_{\varepsilon}-\varepsilon\right)-\sigma_{h}\left(\psi_{\varepsilon}-\varepsilon\right)\right\|_{\mathbb{C}(\bar{\Omega})}=0 .
$$

On conclut, grâce à la normalité de $\mathfrak{C}(\bar{\Omega})$.

H) POTHÉSE DE RÉGULARITF́ : On suppose que pour tout $n$ :

$$
\bar{u}_{n} \cdot \hat{u}_{\|} . u \in W^{1} \cdot(\Omega)
$$

THÉorème 2 : Sous les hypothèses (1), (2), (6) à (9), (19), (30), (36), (38), on a :

$$
\lim _{h \rightarrow 0}\left\|u-u_{h}\right\|_{\mathbb{C}(\bar{\Omega})}=0, \quad \lim _{h \rightarrow 0}\left\|u-u_{h}\right\|_{H^{1}(\Omega)}=0 .
$$

D'après (9), $M \breve{u}_{n}, M \hat{u}_{n} \in W^{1, \infty}$. Pour tout $n$ on utilise alors la proposition 3 et l'estimation (25) de la proposition 1 pour obtenir $\lim _{h \rightarrow 0}\left\|u-u_{h}\right\|_{\mathbb{C}(\bar{\Omega})}=0$.

On en déduit la convergence dans $H^{1}(\Omega)$ fort (cf. [10]).

REMARQUE SUR LES CONDITIONS AUX LIMITES : Il peut être également intéressant de considérer des IQV avec des conditions au bord de type de Dirichlet. On trouvera dans [12] des résultats équivalents à ceux des théorèmes 1 et 2 .

RÉSOLUTION NUMÉRIQUe (calculs effectués sur IRIS $50 \mathrm{CII}$ ) : Pour résoudre numériquement l'IQV discrète, deux points de vue semblent possibles :

1. considérer la solution de l'IQV comme une limite de solution d'IV et résoudre chaque IV par une méthode appropriée (méthode de relaxation, méthode de décomposition, etc.); on s'arrête par exemple lorsque l'écart entre deux IV est assez petit;

2. considérer la solution de l'IQV comme le point fixe d'une méthode mixte : par exemple Miellou a étudié dans [21] le comportement des méthodes mixtes sous-relaxation-algoruhme 1201$)$ ou (iaus-Seidel-algorithme (20). Présentons l'expérimentation numérique suivante. D'après [15], on pose $a_{i i}=1, b_{i}=0, a_{0}=1$ et on considère $h=1 / 18$. On s'est placé dans le cadre d'une méthode de sur-relaxation, la méthode de Gaus-Seidel ayant une convergence lente, tout spécialement dans le cadre de conditions de Neumann. 
$1^{\text {re }}$ expérience : On approxime chaque IV :

test pour une IV : $10^{-5} / 2$;

test entre $2 \mathrm{IV}: 10^{-5} / 2$;

nombre d'IV résolues : 15 ;

nombre total de relaxations : 755 ;

précision : 0,0017 ;

temps : $174,37 \mathrm{~s}$.

$2^{\mathrm{e}}$ expérience : On ne cherche plus à approximer chaque IV en tant que telle et on change l'obstacle toutes les 10 relaxations :

nombre total de relaxations : 430 ;

précision : 0,0017 ;

temps : $93,49 \mathrm{~s}$.

REMARQUE : Grâce à une étude sur la convergence en norme uniforme d'IV, on a pu obtenir des résultats quasi optimaux (cf. [26], [27]).

\section{REMERCIEMENTS}

Je tiens à exprimer ma reconnaissance au professeur J. C. Miellou qui m'a confié cette étude et m'a guidé dans la réalisation de ce travail.

\section{BIBLIOGRAPHIE}

1. C. BAIOcCHI, Estimations d'erreur dans $L^{\infty}$ pour les inéquations à obstacle, Lecture Notes Math. Gem., ${ }^{\circ} 606,1977$, p. 27-34.

2. C. BAIOCCHI et A. CAPELO, Disequazioni variazionali e quasivariazionali. Applicazioni a problemi di frontiera libera, Bologne.

3. C. BAIOCCHI et G. A. Pozzi, Error Estimates and Free Boundary Convergence for a Finite Difference Discretization of a Parabolic Variational Inequality, R.A.I.R.O., vol. $11, n^{\circ} 4,1977$, p. 315-340.

4 A. Bensoussan, M. Goursat et J.-L. Lions, C.R. Acad. Sc., Paris, t. 276, série A, 1973, p. 1279.

5. A. Bensoussan et J.-L. Lions, C.R. Acad. Sc., Paris, t. 276, série A, 1973, p. 1189 et t. 278, série A, 1974, p. 675.

6. A. Bensoussan et J.-L. Lions, Application des inéquations variationnelles en contrôle stochastique, Dunod, Paris.

7. Caffarelli et Friedman, Regularity of the Solution of the Quasi Variational Inequality for the Impulse Control. Part I, Comm. of PDE, t. 3, 1978, p. 745 à 753. Part II, Comm. of PDE, t. 4, 1979, p. 279-291.

8. P. G. Ciarlet, Discrete Maximum Principle for Finite-Difference Operators Aequationes Mathematicae, t, 4, 1970, p. 338-352. 
9 P G Ciarlet et P A Raviart, Maximum principle and uniform convergence for the finite element method Comput methods in appl mech and eng rol 21973 p 1-20

10 P Cortey Dumovi, These de 3 cycle, Besançon 1978

11 P Cortey-Dumont, C R Acad Sc, Paris, t 288, serie A, 1979, p 14

12 P CORTEY-Dumont, Rapports de recherche E R A, no 070654

13 R FALK, Error Estimates for the Approximation of a Class of Var lational Inequalities, Mathematics of computation, vol 28, $\mathrm{n}^{\circ} 128,1974, \mathrm{p}$ 963-971

$14 \mathrm{R}$ Glowinski, $\mathrm{J}-\mathrm{L}$ Lions et $\mathrm{R}$ Tremoliere, Analyse numerique des inequations variationnelles, Dunod, Paris

15 M Goursat et J P Quadrat, Rapport Laboria, $\mathrm{n}^{\circ} 186$

16 B HANNOUZET et $\mathrm{J}$ L JoLy, Methode d'ordre dans l'interpretation de certaines inequatıons variationnelles et applicatıons, Universite de Bordeaux-I

17 B Hannouzet et J L Joly, C R Acad Sc, Paris, t 286, serie A, 1978, p 735

18 T Laetsch, $J$ funct Anal, $n^{\circ} 18,1975, \mathrm{p}$ 286-287

19 J L Menaldi, C R Acad Sc, Paris, t 284, serie A, 1977, p 1499

20 J C Miellou, Cours de D E A , 1974

$21 \mathrm{~J}$ C Miellou, A Mixte Relaxation Algorithm Applied to Quast-Vartational Inequations, Proceedings, 7 th I F I P conference, vol 2, 1975, p 192, SpringerVerlag

$22 \mathrm{~J}$ Nitsche, $L_{\infty}$ Convergence of Finite Element Approximations, Lectures Notes Math Gem, n ${ }^{\circ} 606,1977, \mathrm{p}$ 1-15

23 R Scott, Optimal $L^{\infty}$ Estımates for the Finte Element Method on Irregular, Meshes math of comp, vol 30, $\mathrm{n}^{\circ} 136,1976, \mathrm{p}$ 681-697

24 L Tartar, C R Acad Sc, Paris, t 278, serie A, 1974, p 1193

$25 \mathrm{M}$ Biroli, Estimates in G-Convergence for Varlational and Quast-Variational Inequalities with Bounded Coefficients, Proceeding du Seminaire intensif sur les problemes a frontiere libre, Pavie, octobre 1979 (a paraitre)

26 P Cortey-Dumont, C R Acad Sc, Paris, t 290, ser1e A, 1980, p 255

$27 \mathrm{P}$ Contex-Dumont, On the Approximation of a Class of Quast-Variational Inequalities Related to the Impulse Control, Proceeding du Seminaire intensif sur les problemes a frontiere libre, Pavie, octobre 1979 (a paraître)

28 J Frehse et U Mosco, C R Acad Sc, Paris, t 288, serie A, 1979, p 627

29 E LoInger, Calcolo (a paraître)

30 F Mignot et J P Puel, C R Acad Sc, Paris, t 280, serie A, 1975, p 259

$31 \mathrm{U}$ Mosco, Convergence of Convex Sets and of Solution of Variational Inequalities, Adv Math vol 31969 p 510585

32 U Mosco, Non Linear Quast-Larlational Inequalities and Stochastic Impulse Control theory, Proc conference equadiff IV, Prague 1977, Springer Lecture notes (a paraître)

33 F Brez7i, W W Hager et P A Raviart, Error Estimates for the Finite Element Solution of Variational Inequalities Num Math, vol 28, 1977, p 431 